\title{
Lymphocytes are important in early atherosclerosis
}

\author{
Li Song, ${ }^{1}$ Cynthia Leung, ${ }^{2}$ and Christian Schindler ${ }^{1,2}$ \\ ${ }^{1}$ Department of Medicine, and \\ ${ }^{2}$ Department of Microbiology, Columbia University, New York, New York, USA \\ Address correspondence to: Christian Schindler, HHSC-1212, 701 West 168th Street, New York, New York 10032, USA. \\ Phone: (212) 305-5380; Fax: (212) 543-0063; E-mail: cws4@columbia.edu.
}

Received for publication September 21, 2000, and accepted in revised form May 29, 2001.

\begin{abstract}
Lymphocytes represent a potentially important proinflammatory cell that localizes to atherosclerotic lesions. To determine whether they contribute to lesion development, atherosclerosis-prone ( $\left.L D L R^{-/-}\right)$ mice were crossed with lymphocyte-deficient $\left(R A G 1^{-/-}\right)$mice to generate double knockout progeny. After 8 weeks on a Western-type diet (WTD), lesion development was reduced by $54 \%$ in double knockout mice, as compared with matched $L D L R^{-/-}$controls. However, these significant differences in lesion area gradually subsided as the WTD was continued for 12 and 16 weeks. Consistent with this observation, histological studies determined that lesion initiation and early progression were delayed in $R A G 1 / L D L-R$ double knockout mice. Differences in lesion area did not correlate with any significant alterations in plasma lipid levels. These studies suggest that lymphocytes play an important role early in atherogenesis.
\end{abstract}

J. Clin. Invest. 108:251-259 (2001). DOI:10.1172/JCI200111380.

\section{Introduction}

The localization of both lymphocytes and macrophages to human atheromata suggests that immune response may contribute to the pathogenesis of atherosclerotic lesions (reviewed in refs. 1-3). Detailed characterization of human atheromata has determined that these cells localize to lesions early during pathogenesis; later on they appear to be associated with unstable lesions (2). Previous studies have determined that the Th1 subset of $\mathrm{CD} 4^{+}$ Th cells are the predominate lymphocyte found in atherosclerotic lesions $(1,2,4,5)$. The role Th1 cells play in cellmediated immunity has been well characterized $(6,7)$. Moreover, these cells secrete IFN- $\gamma$, a potent proinflammatory cytokine that induces the expression of major histocompatibility complex (MHC) class II and activation of macrophages $(8,9)$. Consistent with this, lesional macrophages and smooth muscle cells (SMCs) exhibit increased levels of MHC class II expression $(8,10)$. The Th2 subset of $\mathrm{T}$ cells may also contribute to atherosclerosis, but a role for $\mathrm{B}$ cells is less well established $(5,11)$.

With the development of murine models of atherosclerosis (12), it has become possible to more carefully examine the role immune response plays in the development of atherosclerotic lesions. Consistent with the descriptive evaluation of human tissues, studies in mice have highlighted the essential role macrophages play in disease pathogenesis. For example, mice defective in the G-CSF receptor (i.e., low macrophage numbers), MCP-1 (a macrophage-specific chemoattractant), CCR2 (the MCP-1 receptor) and class A or class B scavenger receptors (macrophage lipid receptors) are all defective in atherogenesis (13-20). Likewise, studies on lymphocytes also support a role in atherogenesis. For example, when $\mathrm{CD} 40$ (a potent $\mathrm{T}$ cell activator) is blocked, there is an approximately $60 \%$ reduction in atherosclerosis (21).
When IFN- $\gamma$ (a potent cytokine secreted by T cells) is blocked there is also an approximately $60 \%$ reduction in atherosclerosis (22). Chemokines that are induced by IFN- $\gamma$ have also been implicated in recruitment of T cells to atherosclerotic lesions (23). Likewise, IFN- $\gamma$ has been implicated in the downregulation of $\mathrm{ABC} 1$, a protein that regulates cholesterol efflux from macrophages (24). Functional mutations in $\mathrm{ABC} 1$ have been shown to lead to Tangier disease (25). IFN- $\gamma$ also antagonizes the production of collagen, which is widely believed to stabilize plaque structure $(2,22,26)$. Last, studies on transplant models of atherosclerosis also strongly support a role for IFN- $\gamma$, T cells, and B cells in atherogenesis $(27,28)$. Intriguingly, these studies have demonstrated that $\mathrm{T}$ cell-secreted IFN- $\gamma$ stimulates SMC proliferation through the upregulation of PDGF responses (29). B cells also contribute to transplant atherosclerosis, but their role may be limited to antigen presentation (27).

Several studies have, however, failed to support a role for lymphocytes in atherosclerosis. Two of these studies used lymphocyte-deficient $R A G$-null mice. In one study, cholesterol-fed RAG2/apoE double knockout mice were found to develop lesions at the same rate as "normal" apoE-null mice (30). Similarly, another group found that RAG1/apoE double knockout mice developed atherosclerotic lesions at essentially the same rate as normal apoE-null mice on a high-cholesterol diet (31). Female RAG1/apoE double knockout mice exhibited a modest (i.e., $25 \%$ ), but statistically insignificant, reduction in lesion area. Moreover, when these female mice were evaluated on a chow diet, they exhibited a statistically significant $40 \%$ reduction in lesion area.

To explore this controversy, we selected the $L D L-R$ knockout model of atherogenesis, which appears to be more representative of human disease. $L D L-R$-null 
mice were crossed with the RAG1-null mice, and atherogenesis was evaluated. Although atherosclerotic lesions were small after 4 weeks on a Western type diet (WTD), they had grown significantly by 8 weeks. Moreover, they were $54 \%$ smaller in the RAG1/LDL-R double knockout mice, suggesting lymphocyte function is important at this point in time. Lesions continued to grow with time, but the relative difference in lesion area between $L D L-R$-null and $R A G 1 / L D L$-R double knockout mice became less significant. These results indicate that lymphocytes play a more important role early in the pathogenesis of atherosclerotic lesions.

\section{Methods}

Mice. The $L D L-R$-null mice (in a C57BL/ 6 background) and RAG1-null mice (in a C57BL/6 background) were obtained from The Jackson Laboratory (Bar Harbor, Maine, USA). Mice, maintained in a specific pathogen-free facility, were interbred and genotyped by PCR as described previously (32-34). Once sufficient numbers of single- and double knockout mice were available (i.e., about ten age- and sex-matched mice for each group), they were placed on a WTD ( $21 \% \mathrm{fat}$, $0.15 \%$ cholesterol for 4-16 weeks; Harlan Teklad Laboratory, Winfield, Iowa, USA). Hearts and fasted plasma samples were collected from sacrificed mice.

Histochemistry. Hearts were perfused with PBS, embedded in OCT, and snap-frozen. Then $10-\mu \mathrm{m}$-thick transverse sections covering $350-400 \mu \mathrm{m}$ of the proximal aorta were collected. For atherosclerosis quantitation, every sixth section (for a total of six sections) was stained with Oil red $O$ after fixation in $4 \%$ paraformaldehyde. Lesion area was then determined by measuring accumulated intimal lipid by video microscopy, as described previously (35-37). To evaluate lesion cellularity, nuclei were counted in serial sections that had been stained with Oil red O. Alternating sections were stained with either hematoxylin and eosin (H\&E) or Trichrome after the fixation in 4\% formaldehyde (22). Collagen content was determined by measuring the accumulated collagen in serial Trichrome-stained sections, as described above.

Immunohistochemistry. Cold acetone fixed frozen sections were stained with an MHC-II-specific biotinylated mouse anti-mouse I-A ${ }^{\mathrm{b}}$ antibody (Clone AF6-120.1; PharMingen, San Diego, California, USA). Paraffin sections were stained with rabbit anti-mouse $\alpha$-actin antibody (BioGenex Laboratories, San Ramon, California, USA) or biotinylated anti-F4/80 antibody (a generous gift from A. Stahl), at recommended dilutions, to detect SMCs and macrophages, respectively. Briefly, sections were blocked with horse serum (Vector Laboratories, Burlingame, California, USA) and then incubated with primary antibody for either 1 hour (paraffin sections) or overnight (frozen sections) at room temperature. Endogenous peroxidase was inhibited by ImmunoPure peroxidase suppressor (Pierce Chemical Co., Rockford, Illinois, USA). Sections were then stained with a biotinylated goat anti-rabbit antibody
(Vector Laboratories) and detected with reagents from Vectastain (ABC kit and DAB substrate kit; Vector Labs, Burlingame, California, USA). Sections were counterstained with hematoxylin (Sigma Chemical Co., St. Louis, Missouri, USA) and evaluated under a Nikon Elipse TE300 microscope (Nikon Inc.).

Flow cytometry. Single-cell suspensions were prepared from peripheral blood and stained with an APC-conjugated antibody to CD3 (145-2C11; PharMingen) and a PE-conjugated antibody to CD45R/B220 (RA-3-bB2; PharMingen). All analyses were performed on a FACSCalibur flow cytometer and analyzed with CellQuest software (both from Becton Dickinson and Co., Franklin Lakes, New Jersey, USA).

Lipid studies. Plasma samples, collected at the time of sacrifice, were evaluated for total cholesterol content by a colorimetric enzymatic assay (Cholesterol CII kit; Wako Chemicals USA Inc., Richmond, Virginia, USA). Samples were also pooled and fractionated by FPLC (Superose 6; Amersham Pharmacia Biotech AB, Uppsala, Sweden) prior to evaluation for total cholesterol, as described previously $(35,38)$.

Listeria infection. Listeria monocytogenes were grown and prepared as described previously (39). Before injection, bacteria were diluted to $1 \times 10^{8}$ in sterile PBS and then introduced into the peritoneal cavity. After infection, mice were evaluated twice daily for up to 14 days.

\section{Results}

Generation of RAG1/LDL-R double knockout mice. Previous efforts to examine the role of lymphocytes in the development of atherosclerotic lesions were carried out in the apoE-null model of atherosclerosis. However, studies demonstrating that apoE-null mice exhibit an impaired immune response during infection with Listeria monocytogenes raised a potential concern over using this model to evaluate immune response in atherosclerosis (40).

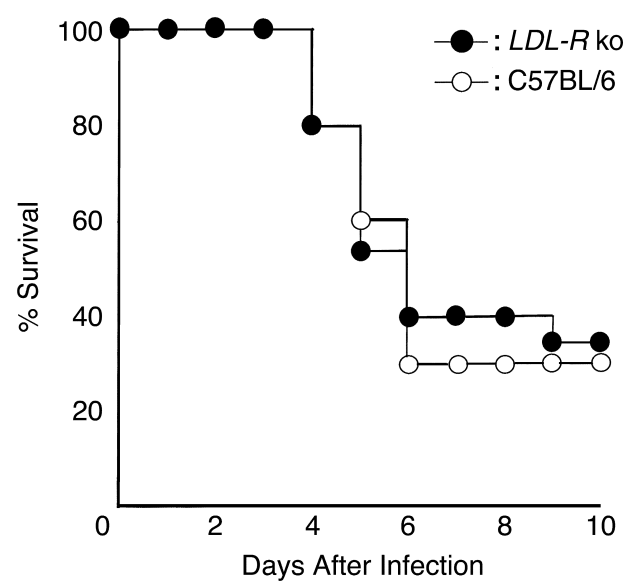

\section{Figure 1}

$L D L-R$ knockout mice exhibit a wild-type survival response to Listeria infection. C57BL/ 6 mice $(n=10)$ and age-matched $L D L-R$ knockout (ko) mice $(n=15)$ were infected intraperitoneally with $1 \times 10^{8} \mathrm{cfu}$ of Listeria monocytogenes. They were monitored twice daily for the following 2 weeks. There were no deaths beyond day 10 . 

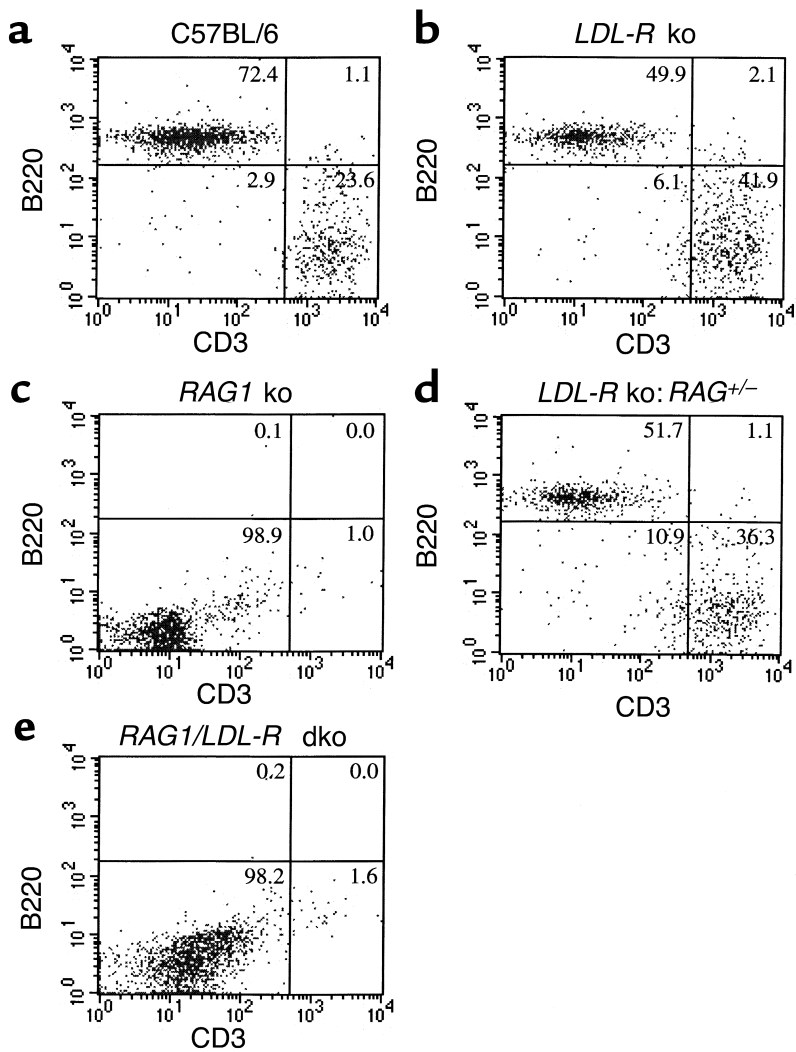

CD3
Figure 2

$R A G 1 / L D L-R$ double knockout ( $\mathrm{dko}$ ) mice do not express peripheral lymphocytes. The number of $\mathrm{CD}^{+}$(a pan T cell marker) and ${\mathrm{B} 220^{+}}^{+}$ (a pan $B$ cell marker) cells found in the peripheral blood of $C 57 \mathrm{BL} / 6$ (a), LDL-R ko (b), RAG1 ko (c), $R A G 1^{+/-}$(d), and $R A G 1 / L D L-R$ dko (e) mice was determined by flow cytometry. These results are representative of more than ten independent experiments.

$\mathrm{mg} / \mathrm{dl}$ ) on a chow diet. In contrast, $L D L-R$-null and the double knockout cholesterol levels were elevated ( 250 $\mathrm{mg} / \mathrm{dl})$ on a chow diet and increased further on a WTD $(\sim 1,300 \mathrm{mg} / \mathrm{dl})$. These studies confirm that the double knockout mice exhibit the elevated cholesterol levels found in $L D L-R$-null mice and the loss of mature lymphocytes found in RAG1-null mice.

Development of atherosclerosis in RAG1/LDL-R double knockout mice. Previous studies in the apoE-null model of atherosclerosis had suggested that lymphocytes have their greatest influence early during murine atherogenesis (31). To examine lesion development in $R A G 1 / L D L$ $R$ double knockout mice, $R A G 1^{+/-} / L D L-\mathrm{R}^{-/-}$and $R A G 1^{-1-} / L D L-R^{-/-}$littermates were placed on a WTD. As shown in Table 2 , mice in both study groups, especially males, gained weight at equivalent rates. Lesion development was then assessed at several time points over a 16-week period of study. At 4 weeks, lesions were extremely modest (i.e., fatty streaks), but smaller (i.e., $\sim 30 \%$ ) in the double knockout mice (Figure 3). By 8 weeks, lesions in both groups had grown considerably and were $54 \%$ smaller $(P<0.001)$ in $R A G 1 / L D L-R$ double knockout mice (Figure 3 and Table 3 ). This signifi-
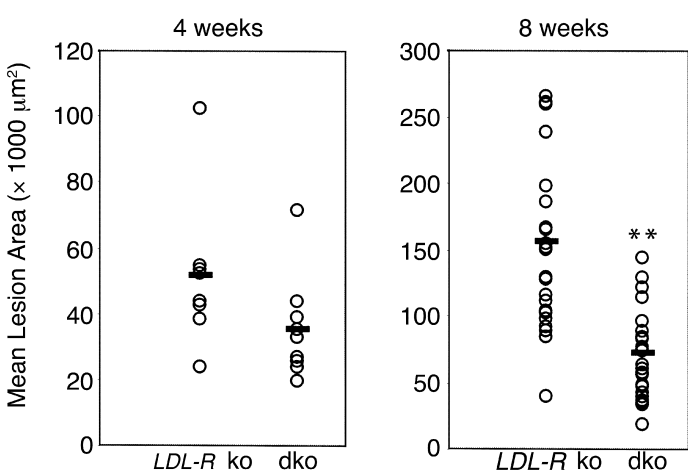

Next, the total plasma cholesterol levels were evaluated in these groups of mice (see Table 1). RAG1 knockout mice exhibited a wild-type level of cholesterol ( $>80$

\section{Figure 3}

The development of atherosclerotic lesions is delayed in $R A G 1 / L D L-R$ double knockout mice. Lesion area from individual $L D L-R$ knockout (ko) and $R A G 1 / L D L-R$ double knockout (dko) mice after 4, 8, 12, and 16 weeks on a WTD are indicated. Data from male and female mice are combined (see Table 3). Mean lesion areas are represented by horizontal bars. The only significant difference between the two study groups is at 8 weeks ( ${ }^{*} P<0.001$; Mann-Whitney $U$ test; Student's $t$ test of either areas or their square roots yielded even lower $P$ values).
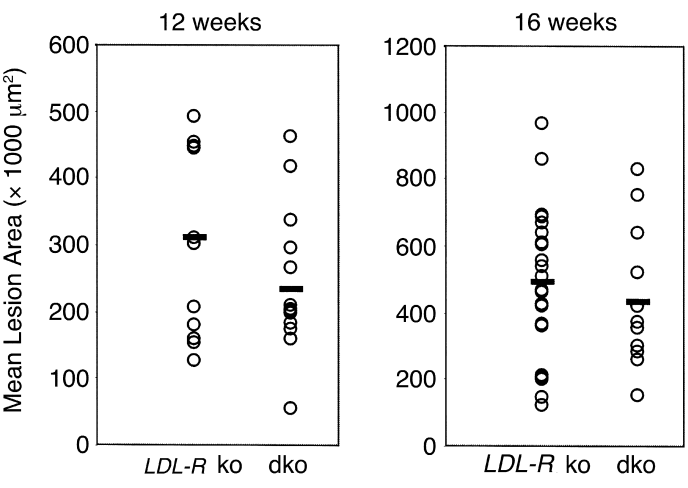

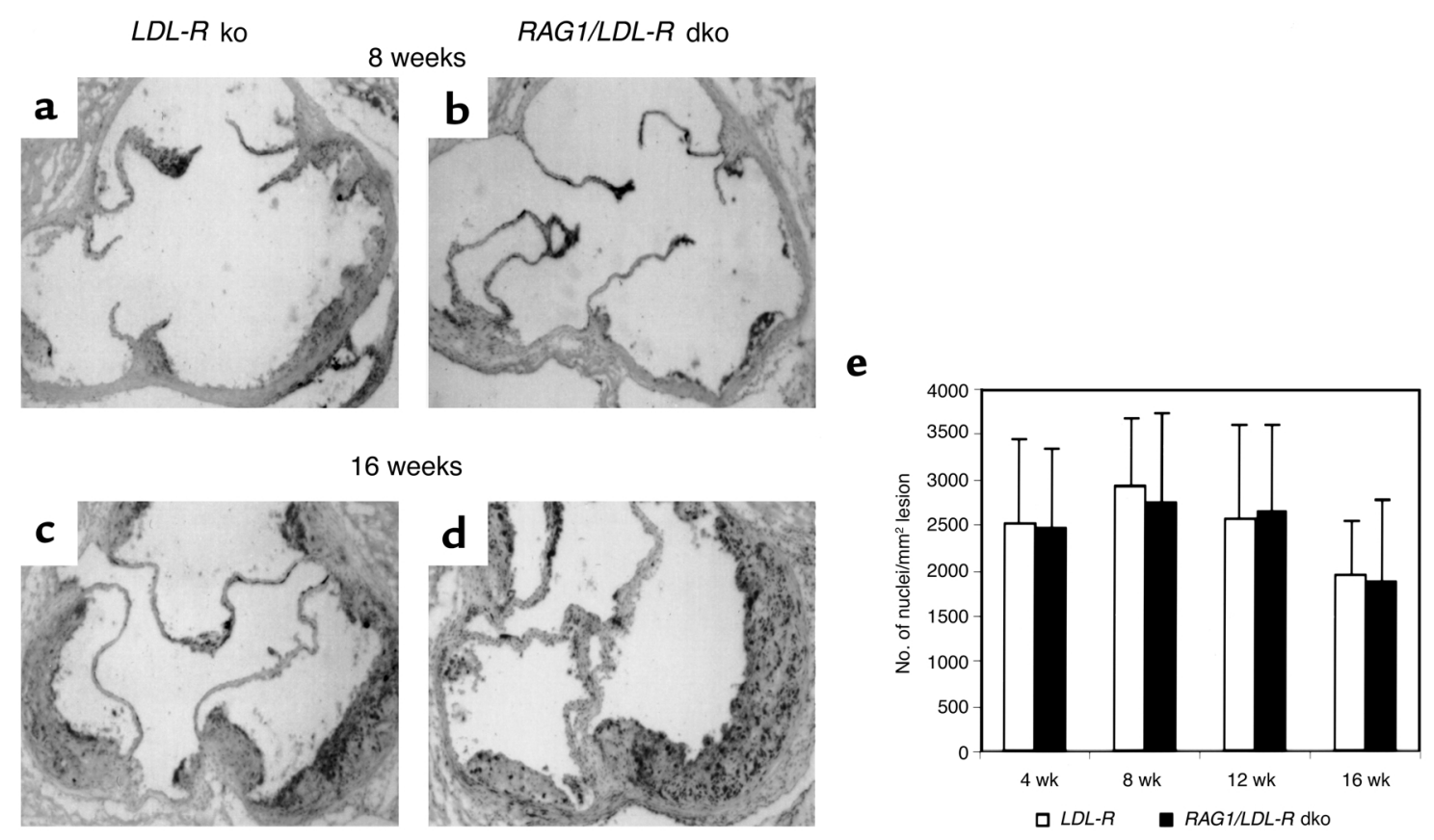

Figure 4

Cell density in atherosclerotic lesions from $L D L-R$ knockout and $R A G 1 / L D L-R$ double knockout mice. Atherosclerotic lesions in the aortic root of LDL-R ko (a and $\mathbf{c}$ ) and $R A G 1 / L D L-R$ dko (b and $\mathbf{d})$ mice, fed a WTD for either 8 weeks ( $\mathbf{a}$ and $\mathbf{b})$ or 16 weeks (c and $\mathbf{d})$, are stained with Oil red $\mathrm{O}$ and hematoxylin (to visualize the nuclei). The average number of nuclei per square millimeter of lesion area, quantitated from serial sections of both study groups at $4,8,12$, and 16 weeks, is presented in $\mathbf{e}$. $L D L-R$ ko mice are represented by open bars, and $R A G 1 / L D L-R$ dko mice by filled bars. Error bars reflect differences in cell density between individual mice in each group. There are no significant differences between study groups.

cant reduction in lesion area was greater in double knockout females (i.e., $62.2 \% ; P \leq 0.017$ ) than in the double knockout males (i.e., $52.5 \%$; $P \leq 0.008$; see Table 3). Lesions also tended to be larger in the females. Although reduced lesion area persisted in the double knockout mice at 12 weeks (i.e., $22 \%$ in males and $32 \%$ in females), this was no longer significant (i.e., $P \leq 0.2$ ). Moreover, differences in lesion area between the $R A G 1 / L D L-R$ double knockout and $L D L-R$ single knockout study groups were virtually absent by 16 weeks of WTD (Figure 3). These observations indicate that lymphocytes play an important role early in atherogenesis.

Histological analysis of atherosclerotic lesions in RAG1/LDL$R$ double knockout mice. To determine whether there were any qualitative differences in the atherosclerotic lesions that developed in $L D L-R$ knockout and $R A G 1 / L D L-R$ double knockout mice, a series of histological studies were carried out. H\&E sections confirmed that lesions

\section{Table 1}

Total plasma cholesterol in C57BL/6, RAG1 ko, $L D L-R$ ko, and $R A G 1 / L D L-R$ dko mice

$\begin{array}{lcccc} & \text { C57BL/6J } & R A G 1 \text { ko } & \text { LDL-R ko } & R A G 1 / L D L-R \text { dko } \\ \text { Chow } & 78 \pm 7 & 78 \pm 6 & 270 \pm 68 & 248 \pm 47 \\ \text { WTD } & 210 & 160 & 1,388 \pm 251 & 1,258 \pm 169\end{array}$

Fasting total cholesterol levels were determined colorimetrically (Cholesterol CII kit; Wako Chemicals USA Inc.) in plasma collected from C57BL/6, RAG1ko, $L D L-R-k o$, and RAG1/LDL-R-dko mice, after 4 weeks on a chow or WTD. were larger and more developed in the RAG1/LDL-R double knockout than in $L D L-R$ knockout mice after 8 weeks of WTD (see Figure 4, a and b). In an effort to characterize these apparent structural differences, two quantitative studies were carried out. First, lesion cellularity was accessed by determining the relative density of nuclei in lesions throughout the study (Figure 4). As anticipated, the steady increase in lesion area observed on WTD correlated with a steady increase in the number of cells (i.e., nuclei) in both study groups (compare Figure 4, a and b, with Figure 4, c and d). However, cellular density within these lesions remained remarkably steady in weeks 4-12 and then dropped modestly by week 16 (Figure 4e). Moreover, there were no significant differences in the lesional cell density between the $L D L-R$ knockout and $R A G 1 / L D L-R$ double knockout mice during the study period.

An increase in collagen content is another important feature in growing atherosclerotic lesions. Previous studies had determined that a loss in the ability to respond to the important $\mathrm{T}$ cell cytokine, IFN- $\gamma$, correlated with an increase in lesion collagen content $(2,22$, 26). A loss of $\mathrm{T}$ cells in $R A G 1 / L D L-R$ double knockout mice might therefore be expected to lead to increased lesional collagen content. To examine this, sections from both study groups were evaluated with Trichrome, which stains collagen blue. As shown in Figure 5, the well-developed lesions found in 8-week $L D L-R$ knockout lesions were rich in collagen content 


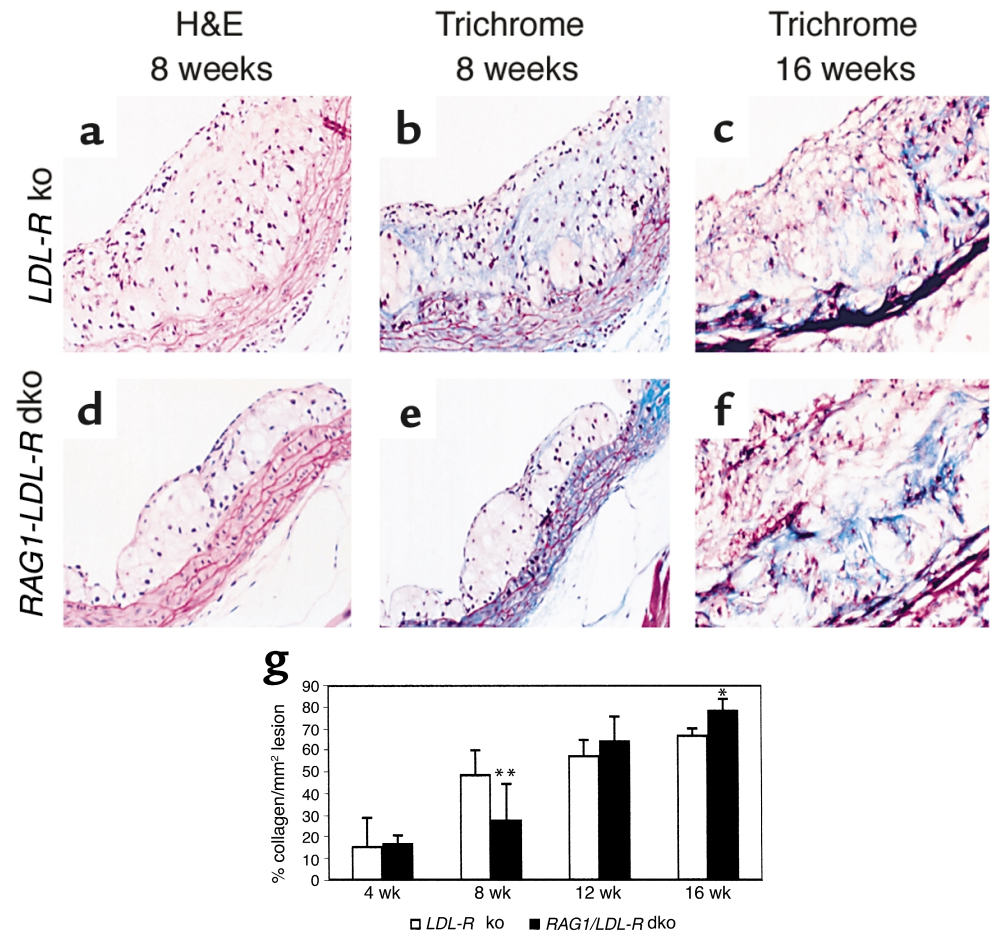

\section{Figure 5}

Collagen contents in the atherosclerotic lesions in $L D L-R$ knockout and $R A G 1 / L D L-R$ double knockout mice. Representative atherosclerotic lesions from the aortic root of $L D L-R$ ko $(\mathbf{a}-\mathbf{c})$ and $R A G 1 / L D L-R$ dko (d-f) mice, fed a WTD for either 8 weeks ( $\mathbf{a}, \mathbf{b}, \mathbf{d}$ and $\mathbf{e})$ or 16 weeks ( $\mathbf{c}$ and $\mathbf{f}$ ) are presented. Specimens were prepared either as paraffin sections ( $\mathbf{a}, \mathbf{b}, \mathbf{d}$ and $\mathbf{e}$ ) or frozen sections ( $\mathbf{c}$ and $\mathbf{f}$ ), and stained either with $\mathrm{H} \& \mathrm{E}$ ( $\mathbf{a}$ and $\mathbf{d}$ ) or Trichrome (b, c, e, and $\mathbf{f}$ ). Collagen, which is stained blue by Trichrome, was quantitated in serial sections in both study groups at $4,8,12$, and 16 weeks. The average collagen content per square millimeter of lesion area is presented in $\mathbf{g}$. $L D L-R$ knockout mice are represented by open bars and $R A G 1 / L D L-R$ dko mice by filled bars. Error bars reflect differences in collagen content between individual mice in each group. Collagen content is significantly lower in dko mice at 8 weeks ( ${ }^{*} P<0.01$, by Mann-Whitney $U$ test) and 16 weeks ( ${ }^{*} P<0.05$, by Mann-Whitney $U$ test).
(Figure $5 \mathrm{~b}$ ). In contrast, the collagen content of the smaller $R A G 1 / L D L-R$ double knockout lesions was considerably more modest (Figure $5 \mathrm{~b}$ with Figure $5 \mathrm{e}$ ). However, by 16 weeks considerably more collagen (and complexity) was evident in the double knockout lesion (Figure 5f). This corresponded with a more modest increase in the $L D L-R$-null lesions (Figure $5 \mathrm{c}$ ). These observations were confirmed by a careful quantitation of the collagen content in serial lesion sections from both study groups (Figure $5 \mathrm{~g}$ ). These studies showed that collagen content was significantly lower at 8 weeks in the $R A G 1 / L D L-R$ double knockout than in the $L D L$ $R$ single knockout lesions $(P<0.01)$. By 12 weeks, the double knockout lesions had caught up and possibly surpassed the $L D L-R$ knockout lesions in collagen content. This trend continued at 16 weeks, but the modest increase in collagen content found in double knockout mice became more significant $(P<0.5)$.

A final set of studies on these lesions entailed immunohistochemistry to evaluate the cell types in these lesions. First, lesions were examined for MHC class II, whose

Table 2

Body weight (grams) in $R A G 1 / L D L-R$ dko and $L D L-R$ ko mice

\begin{tabular}{lcccc} 
Weeks & \multicolumn{2}{c}{ Female } & \multicolumn{2}{c}{ Male } \\
on WTD & LDL-R ko & $R A G 1 / L D L-R$ dko & LDL- $R$ ko & $R A G 1 / L D L-R$ dko \\
4 & $18.4 \pm 1.3(n=4)$ & $17.0 \pm 1.6(n=4)$ & $23.1 \pm 1.1(n=4)$ & $23.4 \pm 2.1(n=5)$ \\
8 & $18.0 \pm 2.0(n=9)$ & $18.0 \pm 2.1(n=10)$ & $24.6 \pm 2.5(n=13)$ & $24.6 \pm 2.3(n=16)$ \\
12 & $18.3 \pm 2.0(n=6)$ & $16.1 \pm 2.9(n=12)$ & $25.3 \pm 4.3(n=7)$ & $21.1 \pm 1.6(n=4)$ \\
16 & $19.5 \pm 3.6(n=8)$ & $21.4 \pm 1.0(n=5)$ & $30.3 \pm 4.9(n=18)$ & $32.5 \pm 7.5(n=7)$
\end{tabular}

Body weights were measured at the time of sacrifice. Numbers of mice in each study group are indicated in parenthesis. Data from male and female mice are shown separately. No significant differences were noted. expression is often considered to reflect a response to IFN- $\gamma(1,41)$. Although MCH class II-positive cells were found scattered throughout lesions (i.e., in macrophages, SMCs, and endothelial cells) in both study groups, they were always more prevalent in $L D L-R$ knockout lesions, suggesting a response to lesional IFN- $\gamma$. A representative analysis of 8-week lesions is shown in Figure 6 (compare Figure $6 \mathrm{a}$ with Figure $6 \mathrm{~b}$ ). Lesions were also stained for a macrophage specific marker. Consistent with evidence from the histological evaluation (see Figures 4 and 5), macrophages were the predominant cell type in the 8week $R A G 1 / L D L-R$ double knockout lesions. The relative decrease in macrophages in $L D L-R$ knockout lesions at 8 weeks (Figure 6, c and d), or later lesions (data not shown), may reflect macrophage apoptosis (42). Staining lesions for $\alpha$-actin revealed a distribution of SMCs that was similar in both $R A G 1 / L D L-R$ double knockout and $L D L-R$ knockout lesions (Figure 6e with Figure 6f). That is, SMCs were predominantly localized to the smooth muscle layer, with a number of positive cells throughout the lesions. These histological studies highlight differences found in early atherosclerotic lesions from $L D L-R$ knockout and $R A G 1 / L D L-R$ double knockout mice.

Plasma cholesterol in

RAG1/LDL-R double knockout mice. During the dietary trial, plasma cholesterol levels of both study groups were carefully measured. Consistent with previous reports $(30,31)$, total plasma cholesterol levels 

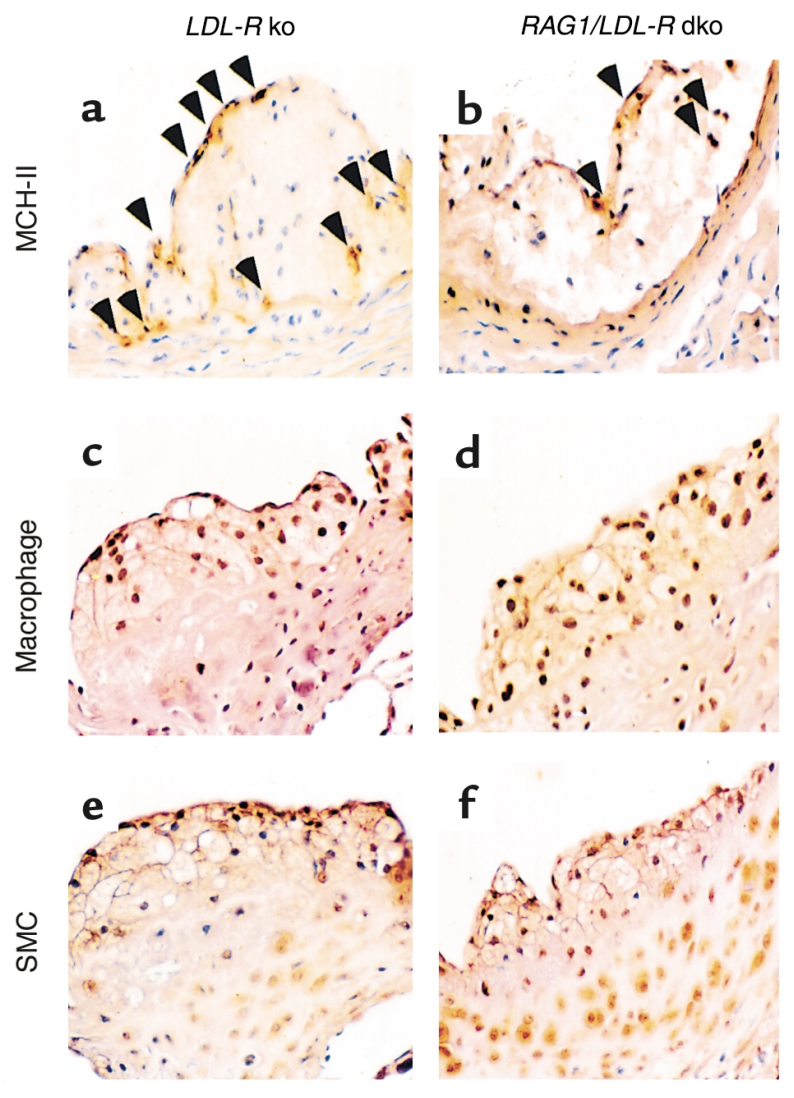

tended to be modestly lower in all of the WTD-fed $R A G$-null mice (Table 4). Although none of these differences were statistically significant, they were more striking in females. Of note, these small differences persisted throughout the study, even when differences in lesion area were rapidly decreasing, and demonstrate a lack of correlation between the differences in cholesterol levels and lesion area. Furthermore, these differences did not correlate with the trend of study mice, especially males, to gain some weight during the study (Table 2).

Next, pooled samples of plasma were fractionated by FPLC chromatography to evaluate lipoprotein fractions $(22,38)$. As anticipated, $L D L-R$-null mice exhibited prominent cholesterol rich VLDL and LDL peaks (see Figure 7). Although the LDL peak, which corre-

\section{Figure 6}

Immunohistochemical analysis of the atherosclerotic lesions in $L D L$ $R$ knockout and $R A G 1 / L D L-R$ double knockout mice. Representative 8-week lesions from $L D L-R$ ko (a, c, and e) and $R A G 1 / L D L-R \mathrm{dko}$ (b, $\mathbf{d}$, and $\mathbf{f}$ ) mice, prepared as frozen sections, are stained with antibodies specific for MHC class II (a and b; AF6-120.1, PharMingen), macrophages (c and d; F4/80), and SMCs (e and f; $\alpha$-actin, BioGenex Laboratories). Positive cells are stained brown (e.g., as indicated with arrows in $\mathbf{a}$ and $\mathbf{b}$ ).

lates more directly with atherosclerosis, remained relatively constant in both groups throughout the study, the VLDL peak in $R A G 1 / L D L-R$ double knockout mice was reduced at 4,8 , and 12 , but not 16 weeks. Buoyant density centrifugation determined that these differences were not due to changes in the levels of apoB and apoA-I (data not shown). It has recently been demonstrated VLDL is considerably less atherogenic than LDL in mice (43). These observations indicate that lymphocytes do not significantly affect the atherogenic profile of plasma lipids. They may, however, influence VLDL metabolism.

\section{Discussion}

The colocalization of activated $\mathrm{T}$ cells and macrophages to early atherosclerotic lesions and to the shoulders of more mature lesions, suggests that cellular immunity may play an important role in the pathophysiology of atherosclerotic disease $(1,3,5,44)$. The development of murine models has provided an opportunity to evaluate the potential role of these immune cells in disease pathogenesis (12). Recent studies in mice have demonstrated that macrophages and two $\mathrm{T}$ cell activators, CD40 and IL-12, effectively promote atherogenesis (13, 21, 45); and that IL-10, a known T cell antagonist, appears to moderate atherogenesis (46-49). Moreover, IFN- $\gamma$, a potent proinflammatory $\mathrm{T}$ cell effector, has also been shown to promote atherogenesis $(2,5,22)$.

A number of observations, however, argue against an important role for $\mathrm{T}$ cells in atherogenesis. There has been a limited success in identifying the antigen(s) that is responsible for activating lesional $\mathrm{T}$ cells. Currently, the most promising antigens appear to be epitopes of oxidized LDL (e.g., malondialdehyde-LDL), which are known to stimulate the formation of auto-antibodies $(1,3,5,50,51)$. Additionally, two studies have reported that atherosclerosis develops normally in lymphocyte-deficient mice $(30,31)$. However, one of these studies noted a $40 \%$ reduction in lesion area when lymphocyte-deficient females were fed a chow diet (31), whereas the sec- 

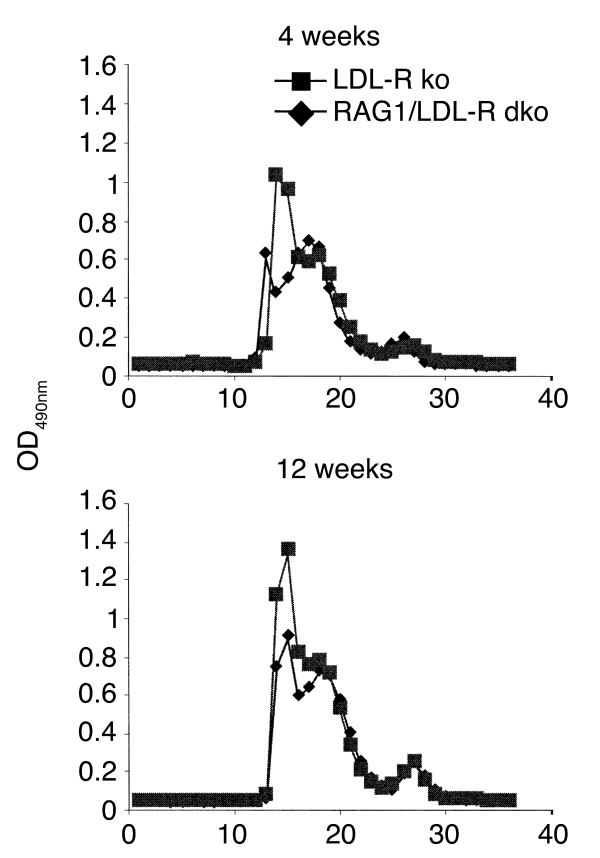
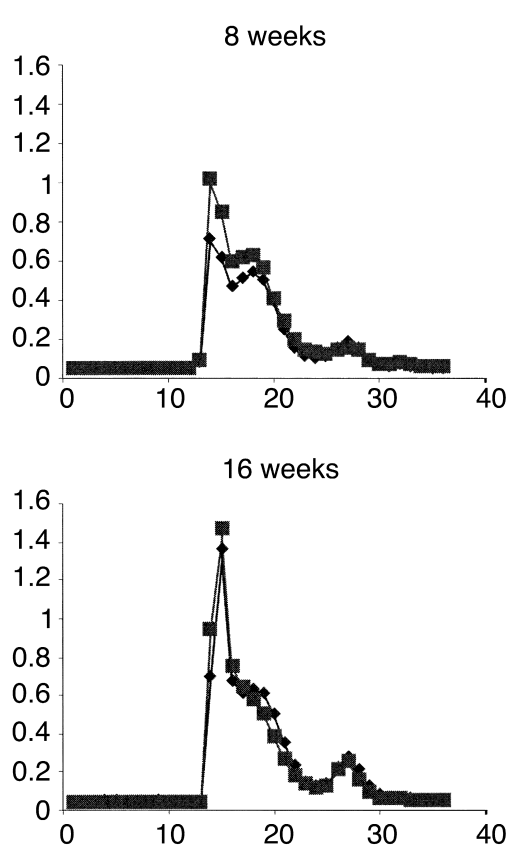

\section{Figure 7}

Plasma cholesterol FPLC profiles. Cholesterol content of pooled plasma collected from $L D L-R$ knockout mice $(n=4)$ and $R A G 1 / L D L-R$ double knockout $(n=4)$ mice fed a WTD for $4,8,12$, and 16 weeks and fractionated by FPLC on a Superose- 6 column, as indicated.

Fractions

ond study focused on more mature lesions (30). This observation suggested that lymphocytes might play a more important role in the early development of atherosclerotic lesions.

Several models have been suggested to account for these conflicting results. For example, lymphocytes may mediate responses that both promote and antagonize atherogenesis. Thus, a loss in lymphocytes might not yield a significant change. In such a scenario, the Th1 subset of T-lymphocytes $(6,7)$, which are implicated in cellular immunity, may serve to promote atherosclerosis. In contrast, Th2 cells, which antagonize Th1 function (7), may serve to impede the development of atherosclerotic lesions. Consistent with this notion, IL-12, a potent Th1 inducer, has been shown to promote atherogenesis $(45,48)$. In contrast, IL-10, a potent Th2 cytokine, has been shown to antagonize atherogenesis (46-49). Another possibility may be that lymphocytes play a more important role early in lesion development, but once a critical level of macrophage recruitment/activation has been achieved, their role

Table 4

Total plasma cholesterol $(\mathrm{mg} / \mathrm{dl})$ in $R A G 1 / L D L-R$ dko and $L D L-R$ ko mice

\begin{tabular}{lcccc}
${ } }$ & \multicolumn{2}{c}{ Female } & \multicolumn{2}{c}{ Male } \\
on WTD & $L D L-R$ ko & $R A G 1 / L D L-R$ dko & $L D L-R$ ko & $R A G 1 / L D L-R$ dko \\
4 & $1,354 \pm 368$ & $1,102 \pm 126$ & $1,229 \pm 279$ & $1,063 \pm 82$ \\
8 & $1,570 \pm 318$ & $1,137 \pm 256$ & $1,454 \pm 315$ & $1,164 \pm 350$ \\
12 & $1,261 \pm 251$ & $1,247 \pm 326$ & $1,351 \pm 271$ & $1,204 \pm 186$ \\
16 & $1,500 \pm 234$ & $1,195 \pm 94$ & $1,427 \pm 476$ & $1,568 \pm 163$ \\
\hline
\end{tabular}

Fasting total cholesterol levels were determined in $L D L-R$ ko and $R A G 1 / L D L-R$ dko mice after $4,8,12$, and 16 weeks on a WTD. Data from male and female mice are shown separately. No significant differences were noted. See Table 2 for the number of mice in each study group. becomes less important. This latter possibility is supported by one study on RAG1/apoE double knockout mice, where the lymphocyte-deficient mice exhibited diminished atherosclerosis on a chow diet (31).

To more directly explore the role of lymphocytes in early atherogenesis, lymphocyte-deficient RAG1 knockout mice were crossed with $L D L-R$ knockout atherosclerosis-prone mice. Of note, we determined that the $L D L-R$ knockout model did not exhibit any potentially confounding perturbations in immune response, as is the case with apoE-null mice (see Figure 1). The $R A G 1 / L D L-R$ double knockout mice developed the anticipated increase in plasma cholesterol when fed a WTD (ref. 52; see Tables 1 and 4). Although these levels tended to be lower than those of RAG1-null mice in each matched group (except the 16-week males), these differences were never statistically significant (Table 4). Similar results have been reported in the RAG1/apoE double knockout mice (31). Consistent with these observations, cholesterol profiles of pooled FPLC fractionated plasma from $R A G 1 / L D L-R$ double knockout mice revealed a notable loss in VLDL cholesterol content (versus $L D L-R$ single knockout mice), except again at 16 weeks. These findings suggest that lymphocytes, or the factors they secrete, have the capacity to regulate VLDL metabolism. This regulatory response could occur either directly in the circulatory system, or in the liver. We favor the latter possibility, as previous studies 
have shown that IL-6, IFN- $\gamma$, and TNF (potentially secreted by large population of lymphocytes found in the GI tract) regulate the liver vitality and metabolism $(22,53,54)$. Differences in cholesterol and apolipoprotein particles did not correlate with differences in atherogenesis (see below).

Comparison of atherosclerotic lesions in $L D L-R$ knockout and $R A G 1 / L D L-R$ double knockout mice identified important differences. Although differences in the small 4-week lesions were not significant, by 8 weeks $R A G 1 / L D L-R$ double knockout lesions were $54 \%$ smaller than $L D L-R$ knockout lesions $(P<0.001)$. This suggested a significant delay in early lesion development. Consistent with previous studies $(30,31)$, this difference subsided over the subsequent weeks of the study. Histological analysis highlighted the lower complexity and smaller size of the lesions found in 8-week $R A G 1 / L D L-R$ double knockout mice. They appeared to consist solely of foam cells, with significantly lower collagen content; again indicating a delay in early lesion progression. In contrast, at 12 weeks collagen content was slightly higher in the RAG1/LDL-R double knockout mice; by 16 weeks this difference had achieved modest significance $(P<0.05)$. This latter observation correlated with our previous finding that collagen content is increased in the atherosclerotic lesions of IFN- $\gamma$ resistant mice (22). That is, because lesional IFN- $\gamma$, which is produced by $\mathrm{T}$ cells, serves to antagonize collagen production $(2,5,10,26)$, then mice without lesional $\mathrm{T}$ cells (i.e., RAG1-null mice) should have increased lesion collagen content. Unexpectedly, we observed only a modest increase in collagen content in mature (i.e., 16-week-old) $R A G 1 / L D L-R$ double knockout lesions. This raised the possibility that other cell types (e.g., macrophages or NK cells; refs. 6, 55) may have contributed to lesional IFN- $\gamma$ production, which in turn antagonized collagen production. Consistent with this possibility, cells in the RAG1/LDL-R double knockout lesions also exhibited modest levels of MHC class II expression at all study time points (Figure 5, and data not shown). However, several other possibilities may account for these unexpected observations. For example, the RAG-null phenotype may lead to a balanced loss of both proatherogenic activities (e.g., IFN- $\gamma$-secreting Th 1 cells; refs. $2,22-25,29)$ and antiatherogenic activities (e.g., IL-10-secreting TH2 cells; refs. 45, 47-49). There may also be inherent differences between collagen accumulation in the apoE knockout model (exploited in earlier studies; refs. 30, 31, 56) and the $L D L-R$ knockout model in the current study. Recent studies indicating that apoE directly antagonize SMC activity support this idea (57). Thus, unique features of the $L D L-R$-null model atherosclerosis may have served to enhance the effect of a loss in lymphocytes on early atherogenesis suggested by an earlier study (31).

The current studies demonstrate that lymphocytes play an important role in the early pathogenesis of atherosclerotic lesions, but with time (e.g., once a critical mass of inflammatory cells has accumulated) other atherogenic stresses compensate for the absence of lymphocytes. One likely stress is the presence of oxidized lipids, which can activate important signaling cascades within macrophages and other lesional cells, and whose presence correlates well with atherogenesis $(51,58-60)$. Oxidized lipids can stimulate the expression of important proinflammatory molecules (e.g., IL-1, TNF, MCP-1, IL-8, CCR2, VCAM, and ICAM-1; ref. 5) and thus promote recruitment and activation of lesional macrophages (61). This pattern of innate responses may, however, be dependent on the high levels of serum lipids found in murine models of atherosclerosis. This may not, however, accurately reflect the events of human atherogenesis, where lesions develop over a more prolonged period. Moreover, in human lesions T cells are predominantly localized to both smaller, less stable lesions and to the more dynamic/pathogenic shoulder region of mature lesions $(2,62)$. Thus, in human lesions $\mathrm{T}$ cells may play a more important role in both lesion development and instability that is associated with acute coronary disease. A more detailed characterization of immune response during early events in murine atherosclerosis is likely to provide important insight into the human atherogenesis.

\section{Acknowledgments}

We thank Alan Tall for many helpful suggestions and critically reading the manuscript. We also thank George Kuriakose for excellent technical assistance. These studies were supported by a grant from the NIH (HL-56984).

1. Hansson, G.K. 1997. Cell-mediated immunity in atherosclerosis. Curr. Opin. Lipidol. 8:301-311.

2. Libby, P. 2000. Changing concepts of atherogenesis. J. Intern. Med. 247:349-358

3. Lichtman, A.H., Cybulsky, M., and Luscinskas, F.W. 1996. Immunology of atherosclerosis: the promise of mouse models. Am. J. Pathol. 149:351-357.

4. Frostegard, J., et al. 1999. Cytokine expression in advanced human atherosclerotic plaques: dominance of pro-inflammatory (Th1) and macrophage-stimulating cytokines. Atherosclerosis. 145:33-43.

5. Glass, C.K., and Witztum, J.L. 2001. Atherosclerosis. The road ahead. Cell. 104:503-516.

6. Abbas, A., Lichtman, A.H., and Pober, J.S. 1991. Cellular and molecular immunology. M.J. Wonsiewicz, editor. W.B. Saunders Co. Philadelphia, Pennsylvania, USA. 225 pp.

7. Murphy, K.M., et al. 2000. Signaling and transcription in T helper development. Annu. Rev. Immunol. 18:451-494.

8. Hansson, G.K., Holm, J., and Jonasson, L. 1989. Detection of activated T lymphocytes in the human atherosclerotic plaque. Am. J. Pathol. 135:169-175.

9. Bach, E.A., Aguet, M., and Schreiber, R.D. 1997. The IFN gamma receptor: a paradigm for cytokine receptor signaling. Annu. Rev. Immunol. 15:563-591.

10. Libby, P. 1995. Molecular bases of the acute coronary syndromes. Circulation. 91:2844-2850.

11. Zhou, X., Paulsson, G., Stemme, S., and Hansson, G.K. 1998. Hypercholesterolemia is associated with a T-helper (TH) $1 / \mathrm{TH} 2$ switch of the autoimmune response in atherosclerotic apo E-knockout mice. J. Clin. Invest. 101:1717-1725.

12. Breslow, J.L. 1996. Mouse model of atherosclerosis. Science. 272:685-688.

13. Smith, J.D., et al. 1995. Decreased atherosclerosis in mice deficient in both macrophage colony-stimulating factor (op) and apolipoprotein E. Proc. Natl. Acad. Sci. USA. 92:8264-8268.

14. Gu, L., et al. 1998. Absence of monocyte chemoattractant Protein-1 reduces atherosclerosis in low density lipoprotein receptor deficient mice. Mol. Cell. 2:275-281.

15. Gosling, J., et al. 1999. MCP-1 deficiency reduces susceptibility to atherosclerosis in mice that overexpress human apolipoprotein B. J. Clin. Invest. 103:773-778.

16. Boring, L., Gosling, J., Cleary, M., and Charo, I.F. 1998. Decreased lesion 
formation in CCR2 -/- mice reveals a role for chemokines in the initiation of atherosclerosis. Nature. 394:894-897.

17. Febbraio, M., et al. 2000. Targeted disruption of the class B scavenger receptor CD36 protects against atherosclerotic lesion development in mice. J. Clin. Invest. 105:1049-1056.

18. de Winther, M.P., et al. 1999. Scavenger receptor deficiency leads to more complex atherosclerotic lesions in APOE3Leiden transgenic mice. Atherosclerosis. 144:315-321.

19. Suzuki, H., et al. 1997. A role for macrophage scavenger receptors in atherosclerosis and susceptibility to infection. Nature. 386:292-296.

20. Sakaguchi, H., et al. 1998. Role of macrophage scavenger receptors in diet-induced atherosclerosis in mice. Lab. Invest. 78:423-434.

21. Mach, F., Schonbeck, U., Sukhova, G.K., Atkinson, E., and Libby, P. 1998. Reduction of atherosclerosis in mice by inhibition of CD40 signaling. Nature. 394:200-203.

22. Gupta, S., et al. 1997. IFN- $\gamma$ potentiates atherosclerosis in apoE knockout mice. J. Clin. Invest. 99:2752-2761.

23. Mach, F., et al. 1999. Differential expression of three T lymphocyte-activating CXC chemokines by human atheroma-associated cells. J. Clin. Invest. 104:1041-1050.

24. Panousis, C.G., and Zuckerman, S.H. 2000. Interferon-gamma induces downregulation of Tangier disease gene (ATP- binding-cassette transporter 1) in macrophage-derived foam cells. Arterioscler. Thromb. Vasc. Biol. 20: $1565-1571$

25. Tall, A.R., and Schindler, C.W. 2000. A, B, C...gamma! Arterioscler. Thromb. Vasc. Biol. 20:1423-1424.

26. Rekhter, M.D., et al. 1993. Type I collagen gene expression in human atherosclerosis. Localization to specific plaque regions. Am. J. Pathol. 143:1634-1648.

27. Shi, C., et al. 1999. Donor MHC and adhesion molecules in transplant arteriosclerosis. J. Clin. Invest. 103:469-474.

28. Nagano, H., et al. 1997. Interferon- $\gamma$ deficiency prevents coronary atherosclerosis but not myocardial rejection in transplanted mouse hearts. J. Clin. Invest. 100:550-557.

29. Tellides, G., et al. 2000. Interferon-gamma elicits arteriosclerosis in the absence of leukocytes. Nature. 403:207-211.

30. Daugherty, A., et al. 1997. The effects of total lymphocyte deficiency on the extent of atherosclerosis in apolipoprotein $\mathrm{E}-/-$ mice. J. Clin. Invest. 100:1575-1580.

31. Dansky, H.M., Charlton, S.A., McGee-Harper, M., and Smith, J.D. 1997. $\mathrm{T}$ and $\mathrm{B}$ lymphocytes play a minor role in atherosclerotic plaque formation in the apolipoprotein E-deficient mouse. Proc. Natl. Acad. Sci. USA. 94:4642-4646.

32. Ishibashi, S., Goldstein, J.L., Brown, M.S., Herz, J., and Burns, D.K. 1994. Massive xanthomatosis and atherosclerosis in cholesterol-fed low density lipoprotein receptor-negative mice. J. Clin. Invest. 93:1885-1893.

33. Abrahamsohn, I.A., and Coffman, R.L. 1996. Trypanosoma cruzi: IL-10, TNF, IFN- $\gamma$ and IL-12 regulate mice innate and acquired immunity to infection. Exp. Parasitol. 84:231-244.

34. Mombaerts, P., et al. 1992. RAG-1-deficient mice have no mature B and T lymphocytes. Cell. 68:869-877.

35. Plump, A.S., et al. 1992. Severe hypercholesterolemia and atherosclerosis in apolipoprotein E-deficient mice created by homologous recombination in ES cells. Cell. 71:343-353.

36. Paigen, B., Morrow, A., Holmes, P.A., Mitchell, D., and Williams, R.A. 1987. Quantitative assessment of atherosclerotic lesions in mice. Ather osclerosis. 68:231-240.

37. Rubin, E.M., Krauss, R.M., Spangler, E.A., Verstuyft, J.G., and Clift, S.M. 1991. Inhibition of early atherogenesis in transgenic mice by human apolipoprotein AI. Nature. 353:265-267.

38. Homanics, G.E., et al. 1995. Mild dyslipidemia in mice following targeted inactivation of the Hepatic Lipase gene. J. Biol. Chem. 270:2974-2980.

39. Harty, J.T., and Bevan, M.J. 1995. Specific immunity to Listeria monocytogenes in the absence of IFN- $\gamma$. Immunity. 3:109-117.

40. Roselaar, S.E., and Daugherty, A. 1998. Apolipoprotein E-deficient mice have impaired innate immune responses to Listeria monocytogenes in vivo. J. Lipid Res. 39:1740-1743

41. Hansson, G.K., Hellstraand, M., Rymo, L., Rubbia, L., and Gabbiani, G. 1989. Interferon gamma inhibits both proliferation and expression of differentiation-specific alpha-smooth muscle actin in arterial smooth muscle cells. J. Exp. Med. 170:1595-1608.

42. Bjorkerud, S., and Bjorkerud, B. 1996. Apoptosis is abundant in human atherosclerotic lesions, especially in inflammatory cells (macrophages and $\mathrm{T}$ cells), and may contribute to the accumulation of gruel and plaque instability. Am. J. Pathol. 149:367-380.

43. Veniant, M.M., et al. 2000. Defining the atherogenicity of large and small lipoproteins containing apolipoprotein B100. J. Clin. Invest. 106:1501-1510.

44. Libby, P., and Hansson, G.K. 1991. Involvement of the immune system in human atherogenesis: current knowledge and unanswered questions. Lab. Invest. 64:5-15.

45. Lee, T.S., Yen, H.C., Pan, C.C., and Chau, L.Y. 1999. The role of interleukin 12 in the development of atherosclerosis in ApoE-deficient mice. Arterioscler. Thromb. Vasc. Biol. 19:734-742.

46. Mallat, Z., et al. 1999. Protective role of interleukin-10 in atherosclerosis. Circ. Res. 85:E17-E24.

47. Pinderski Oslund, L.J., et al. 1999. Interleukin-10 blocks atherosclerotic events in vitro and in vivo. Arterioscler. Thromb. Vasc. Biol. 19:2847-2853.

48. Uyemura, K., et al. 1996. Cross-regulatory roles of interleukin (IL)-12 and IL-10 in atherosclerosis. J. Clin. Invest. 97:2130-2138.

49. Terkeltaub, R.A. 1999. IL-10: an "immunologic scalpel" for atherosclerosis? Arterioscler. Thromb. Vasc. Biol. 19:2823-2825.

50. Witztum, J.L., and Berliner, J.A. 1998. Oxidized phospholipids and isoprostanes in atherosclerosis. Curr. Opin. Lipidol. 9:441-448.

51. Witztum, J.L. 1994. The oxidation hypothesis of atherosclerosis. Lancet 344:793-795

52. Ishibashi, S., Goldstein, J.L., Brown, M.S., Herz, J., and Burns, D.K. 1994 Massive xanthomatosis and atherosclerosis in cholesterol-fed low density lipoprotein receptor-negative mice. J. Clin. Invest. 93:1885-1893.

53. Cressman, D.W., et al. 1996. Liver failure and defective hepatocyte regeneration in interleukin-6-deficient mice. Science. 274:1379-1383.

54. Li, Q., Van Antwerp, D., Mercurio, F., Lee, K.F., and Verma, I.M. 1999. Severe liver degeneration in mice lacking the IkappaB kinase 2 gene. Science. 284:321-325.

55. Munder, M., Mallo, M., Eichmann, K., and Modolell, M. 1998. Murine macrophages secrete interferon gamma upon combined stimulation with interleukin (IL)-12 and IL-18: A novel pathway of autocrine macrophage activation. J. Exp. Med. 187:2103-2108.

56. Gupta, S., et al. 1996. The SH2 domains of Stat 1 and Stat 2 mediate multiple interactions in the transduction of IFN- $\alpha$ signals. EMBO J. 15:1075-1084

57. Ishigami, M., Swertfeger, D.K., Hui, M.S., Granholm, N.A., and Hui, D.Y. 2000. Apolipoprotein $\mathrm{E}$ inhibition of vascular smooth muscle cell proliferation but not the inhibition of migration is mediated through activation of inducible nitric oxide synthase. Arterioscler. Thromb. Vasc. Biol. 20:1020-1026.

58. Peet, D.J., et al. 1998. Cholesterol and bile acid metabolism are impaired in mice lacking the nuclear oxysterol receptor LXR $\alpha$. Cell. 93:693-704.

59. Shih, D.M., et al. 1998. Mice lacking serum paraoxonase are susceptible to organophosphate toxicity and atherosclerosis. Nature. 394:284-287.

60. Mahley, R.W., Weisgraber, K.H., Innerarity, T.L., and Rall, S.C. 1991. Genetic defects in lipoprotein metabolism: elevation of atherogenic lipoproteins caused by impaired catabolism. JAMA. 265:78-83.

61. Chang, M.K., et al. 1999. Monoclonal antibodies against oxidized lowdensity lipoprotein bind to apoptotic cells and inhibit their phagocytosis by elicited macrophages: evidence that oxidation-specific epitopes mediate macrophage recognition. Proc. Natl. Acad. Sci. USA. 96:6353-6358.

62. van der Wal, A.C., Becker, A.E., van der Loos, C.M., and Das, P.K. 1994. Site of intimal rupture or erosion of thrombosed coronary atherosclerotic plaques is characterized by an inflammatory process irrespective of the dominant plaque morphology. Circulation. 89:36-44. 